\title{
Research Paper \\ Predicting Elderly Depression Prevalence in Different Iranian Ethnicities and Associated Factors
}

\author{
*Elahe Allahyari ${ }^{1} \mathbb{\oplus}$, Majid Keramati², Mohammadreza Kamali
}

1. Department of Epidemiology and Biostatistics, Social Determinants of Health Research Center, Faculty of Health, Birjand University of Medical Sciences, Birjand, Iran 2. Department of Public Health, Student Research Committee, Birjand University of Medical Sciences, Birjand, Iran.

\begin{tabular}{l|l}
$\begin{array}{c}\text { Use your device to scan } \\
\text { and read the artice online }\end{array}$ \\
Associated Factors (Persian)]. Iranian Journal of Ageing. 2020; 15(1):94-103. https://doi.org/10.32598/sija.2020.3.130 \\
doi https://doi.org/10.32598/sija.2020.3.130
\end{tabular}

\section{(i) (8)}

Received: 13 Dec 2018 Accepted: 24 Jun 2019 Available Online: 01 Apr 2020

Key words: Elderly, Depression, Prevalence, Associated factors, Ethnicity

\section{A B STRACT}

Objectives There is currently a lack of consensus to estimate and compare depression proportion of elderly people in different Iranian ethnicities. Therefore, we examined the effect of ethnicity on elderly depression in the presence of other variables.

Methods \& Materials During a cross-sectional study in 2017, seven Iranian ethnicities were considered as clusters, then a random sample was selected from each group of people over 60 years old. A total of 1477 participants were studied among which 675 were Fars, 298 Turk, 100 Kurds and Turkman, 99 Baloch and Arab, and 96 Lor. The subjects were asked to complete the Elderly Depression Inventory (GDS-15) and demographic checklist. Data were analyzed by SPSS V. 22 software in $\alpha=0.05$ using linear regression analysis and ANOVA test.

Results The prevalence of depression was only about $50 \%$. However, depression proportion of Baluch, Arab, Kurd and Lor elderlies with 81.8, 77.8, 62, and 59.4 percent was significantly high. Even, the prevalence of severe depression was $30 \%$ in Baluchis. Also, linear regression revealed that widowhood $(P<0.001)$, retirement $(P=0.022)$, house bound $(P<0.001)$, and the increasing chronic illnesses $(P<0.001)$, and income $(P<0.001)$ were other variables influencing depression.

Conclusion The establishment of methods to decreasing elderly depression is necessary because of the high prevalence of depression among the elderly of different ethnicities, especially the Arab and Baluch ethnic groups.

\section{Extended Abstract}

\section{Introduction}

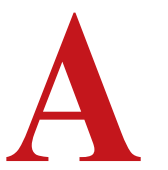

ging is an inevitable physiological and physical phenomenon. The same as other countries, the Iranian population is also becoming old. The statistics show that from 1957 to 2007, Iran's elderly popula- tion rose almost 4.4 times, but the total population in the same period increased only 7.3 times. Consequently, Iran will also face the problem of an elderly population similar to other Asian countries in the near future. Thus the rise of the elderly population will be a major issue in economic, societal, and health care in the $21^{\text {st }}$ century. Consideration of the geriatric, and their provision, protection and promotion of their mental health is of high significance.

* Corresponding Author:

Elahe Allahyari, PhD.

Address: Department of Epidemiology and Biostatistics, Social Determinants of Health Research Center, Faculty of Health, Birjand University of Medical Sciences, Birjand, Iran.

Tel: +98 (56) 32381661

E-mail: elaheh.allahyari@gmail.com 
Depression is a very common disorder in the old ages, which is caused by some various factors associated with this stage of life. But an important point that will be effective for the health policy-making and educational/training programs in order to alleviate the said problem is awareness of the depression level and its concentration in different regions of our country, which is due to the fact that the population concentration in Iran depends on the different races.

This study aims to measure the level of depression prevalence in different Iranian races. Therefore, not only the information required for programming on the alleviation of this problem will be provided, but also, regarding the different cultures and traditions of these races, such data will be regarded as the foundation for analyzing the impacts of different dimensions of this factor on the depression of the elderlies.

\section{Methods \& Materials}

Yesavage originally developed the GDS questionnaire in a set of 30 items. This questionnaire asked the people to answer the questions due to their feelings during past week. In 1986, the 15 question form of the questionnaire, including yes $=1$ and no $=1$ answers was provided. However, items 1,5 , 7,11 and 13 in this questionnaire were scored differently and the "no" answer receives the score of 1 . And the sum of the scores forms the depression score of each person. The scores show low, moderate and severe depression when those were between 5 and 8, 9 and 11, and above 12, respectively.

In 2006, the Persian version of this questionnaire was prepared through translation, inverse translation, and matching them. The Persian 15-item questionnaire had also high Cronbach's Alpha (0.9), split-half (0.98), and the test-retest (58.8). According to the demographic composition of Iran in 2001, Persians and Turkic races form two major components of Iran's population with respectively 51 and 24 . So, our study considered these population proportions and collected a sufficient sample from seven Iranian races: the Persian, Turkic, Kurd, Lur, Baloch, Arab, and Turkman races.

Initially, they were provided with the required explanations about the purpose of the study, and then the informed consent was obtained from those being a volunteer to participate in the study. The subjects were requested to fill in the GDS questionnaire. If the interviewees were illiterate, the researcher completed the questionnaire during the interview. After removing incomplete questionnaires, the information were analyzed by SPSS V. 22 software.

\section{Result}

The present research studied 1477 people between 6092 years old. In general, $56.9 \%$ of the subjects were male and $44.5 \%$ of people were residents of the rural areas. The Chi-square test shows a significant relationship between

Table 1. The regression analysis for the impact of different variables on their depression.

\begin{tabular}{|c|c|c|c|}
\hline Variables & Standardized Betaa & t-test Statisticb & Pc \\
\hline Age (y) & 0.038 & 1.43 & 0.154 \\
\hline Marital status (reference Married) & 0.127 & 4.61 & $<0.001$ \\
\hline Family members & -0.041 & -1.49 & 0.137 \\
\hline Income & -0.149 & -5.58 & $<0.001$ \\
\hline Job (reference working) & 0.059 & 2.29 & 0.022 \\
\hline Staying at home (reference no) & 0.127 & 5.12 & $<0.001$ \\
\hline Gender (reference Male) & 0.023 & 0.88 & 0.380 \\
\hline Residents (reference Rural) & 0.043 & 1.66 & 0.098 \\
\hline Number of chronic diseases & 0.162 & 6.51 & $<0.001$ \\
\hline \multicolumn{4}{|c|}{ Ethnic (reference Persian) } \\
\hline Turk & 0.018 & 0.73 & 0.466 \\
\hline Kurd & 0.111 & 4.46 & $<0.001$ \\
\hline Lur & 0.107 & 4.23 & $<0.001$ \\
\hline Baloch & 0.165 & 6.27 & $<0.001$ \\
\hline Arab & 0.080 & 2.92 & 0.004 \\
\hline Turkmen & 0.013 & 0.54 & 0.589 \\
\hline
\end{tabular}


the depression severity level and ethnicity in $\operatorname{Iran}(\mathrm{P}<0.001$, $\mathrm{X} 2=197.14)$. As the Table 1 shows, the proportion of the depression severity level experience decreasing trend in all three Persians, Turkic, and Lurs. About half of them show no sign of depression (54.6\% for Persians, $50 \%$ for Turkic, and $41 \%$ for Lur). In Persians and Turkic, the proportion of those suffering from low depression is about half of those having no depression. In none of these three races, the prevalence of moderate depression was no higher than $21 \%$ and the prevalence of severe depression was no higher than $8 \%$.

In Lur and Turkmen races, the proportion of no-depressed people was approximately equal to low depression ones, but the depression above the moderate level is yet below $21 \%$ and in Turkmen even severe depression disappeared. Among Arabs, though the prevalence of the low depression is two times more than those not depressed, only $29 \%$ of subjects in this race suffer from moderate and severe levels of depression. Nonetheless, the situation is critical among the Baloch who only 18.2 percent of them are not depressed, and other $81.8 \%$ show some levels of depression $(24.2 \%$ low, $27.3 \%$ moderate, and $30.3 \%$ severe depression).

The dead couple of subjects were significantly more depressed than married counterparts $(\mathrm{P}<0.001$, Table 1$)$. The level of income, occupation, unemployment, and chronic diseases are other variables affect depression. Depression in Turkic and Turkmen individuals weren't significantly different from Persian ones, but the depression rates in Kurd, Lur, Baloch, and Arab were 0.111, 0.107, 0.165 and 0.08 higher than Persian, respectively. However, gender, living place, age, and familial relationship with other people couldn't significantly change the depression scores.

\section{Conclusion}

In accordance with the findings of the present study and past research, the government is required to make some major policies on reducing the depression in the whole country, especially for Baloch, Arabs, and Turkmen races and females. the growing rate of depression among the Arabs and Turkmen races and the $81.8 \%$ prevalence rate of depression among Baloch elderlies is alarming. Also, female suffered more by depression than men. Examining the variables impacting the depression of the elderly geriatric, due to the fact that those elderly with deceased spouse suffer from more (severe) depression, thus, some special attention to this group requires periodic screening and special education for their families. Another factor deserves attention in the national planning and general policy-making is the income of the elderly. Senior retirees should be engaged in social affairs and group plans and they should refrain from isolation. Creating a happy place for the elderly so that they pass their time there can also be effective.

\section{Ethical Considerations}

\section{Compliance with ethical guidelines}

All ethical principles are considered in this article. The participants were informed about the purpose of the research and its implementation stages; they were also assured about the confidentiality of their information; moreover, they were free to leave the study whenever they wished, and if desired, the research results would be available to them. Also, this study was approved by the Ethics Committee of Birjand University of Medical Sciences (Code: IR.BUMS.REC.1396.226).

\section{Funding}

This work was supported by Birjand University of Medical Sciences under Grant Number 96-4414.

\section{Authors' contributions}

Conceptualization, Investigation, Resources, Writing original draft preparation, Funding acquisition: All author; Methodology, Writing-review \& editing, formal analysis, data curation, supervision: Elahe Allahyari.

\section{Conflicts of interest}

The authors declared no conflict of interest.

\section{Acknowledgements}

The researchers would like to appreciate Dr. Torabi, Dr. Sedarat, Mr. Shirani, Mr. Shokouhi, Mr. Mohammadali Barati, Mr. Meysam Daghlavi, Mr. Ayyub Rezazadeh, Ms. Garnjiak Who have helped us preparing this article. 


\title{
تعيين شيوع افسردكى در سالمندان قوميتهاى مختلف ايرانى و عوامل بيشبينى كننده آن
}

\author{
"الهه اللهيارى'1ه، مجيد كرامتى'، محمدرضا كمالى'

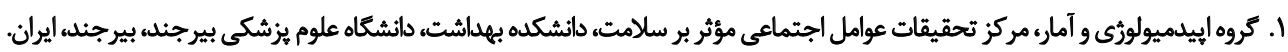

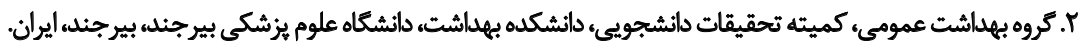

\begin{abstract}
حكSد

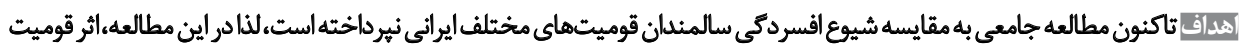

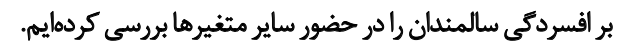

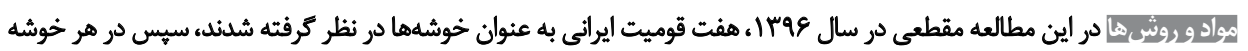

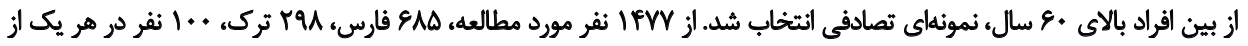

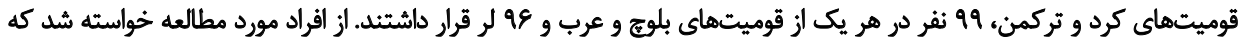

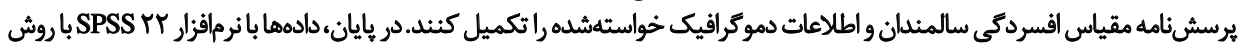

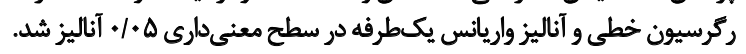

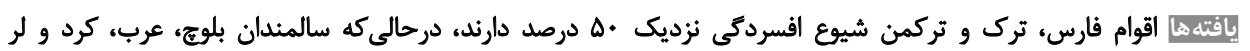

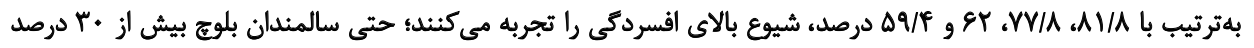

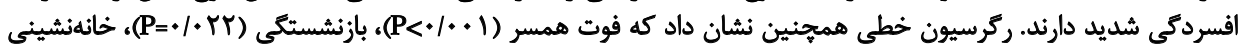

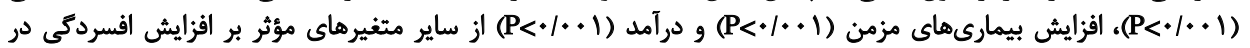
سالمندان است.

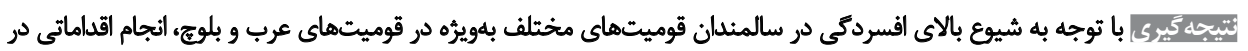
راستاى افزايش نشاط در سالمئدان ضرورى به به نظر مىرسد.
\end{abstract}

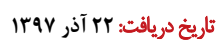

تاريخ هذيرش: بـ ت تير

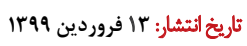

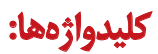

سالمنده افسردكى،شيوع، عوامل مؤثر، قوميت أنيت

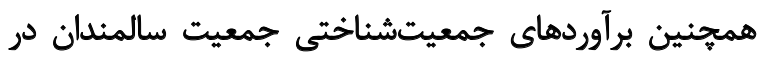

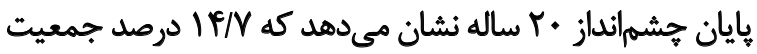

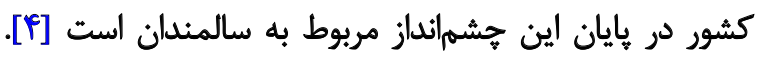

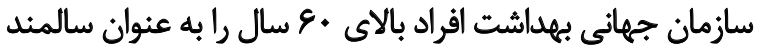

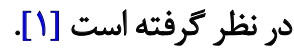

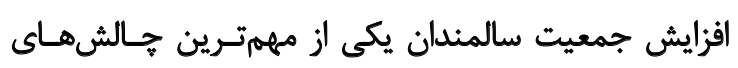

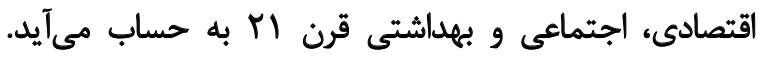

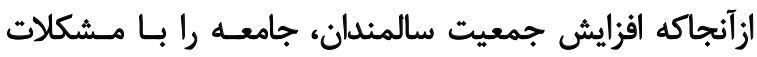

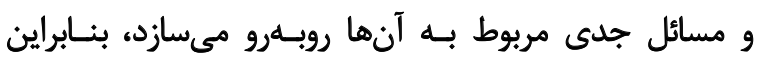

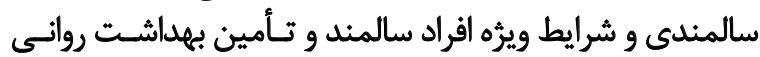

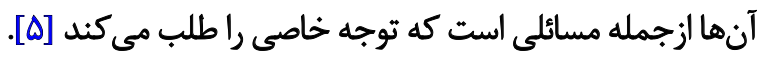

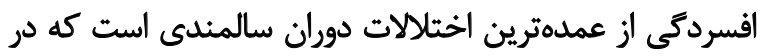

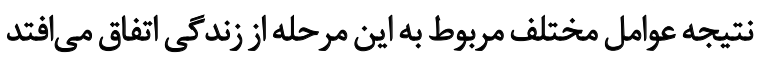

مقدمه

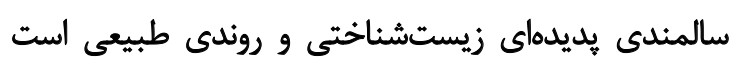

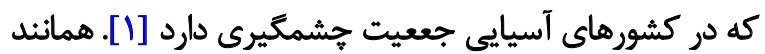

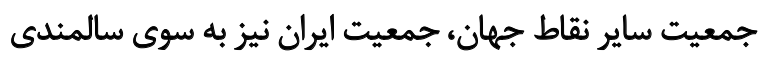

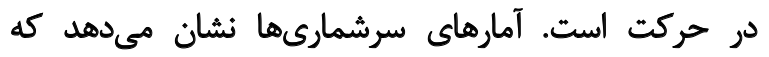

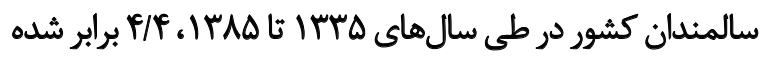

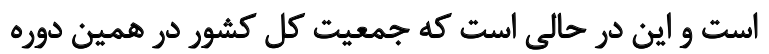

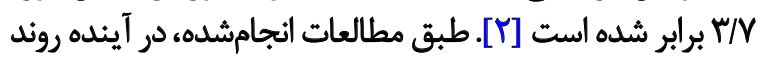

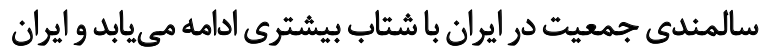

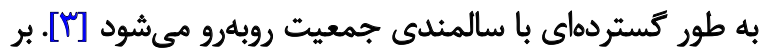

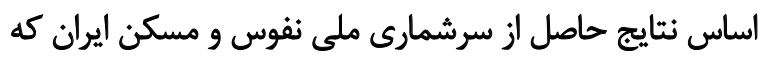

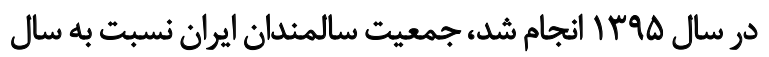

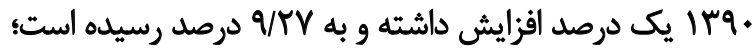


خاص يا سوك عزيزان نشده بودند، انتخاب شدند. در ابتداي

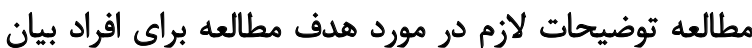

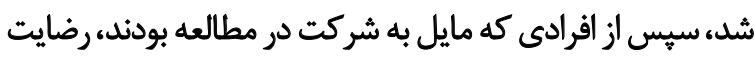

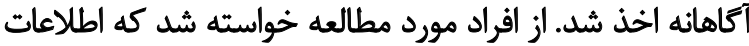

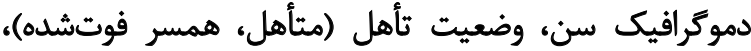

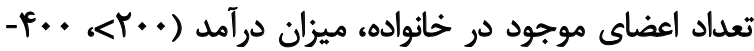

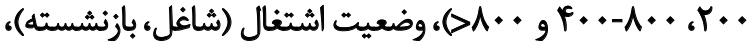

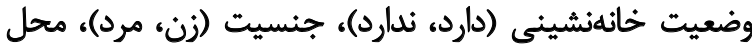

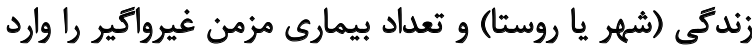

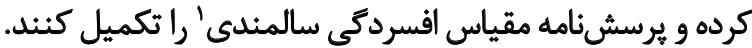

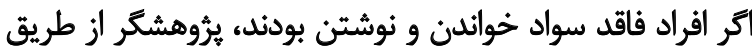

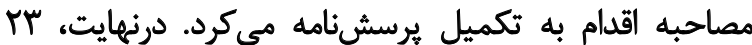

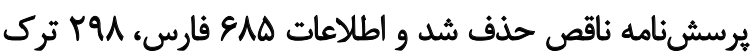

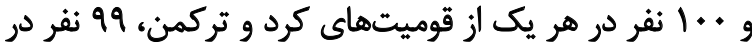

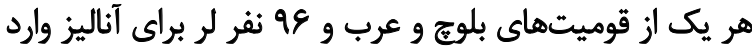

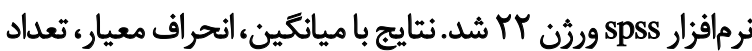

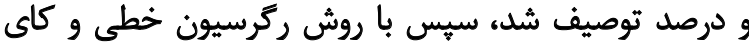
اسكوئر در سطح هـ • آ آناليز شد.

$$
\text { يرسش نامه مثياس افسرديّى سالمثلى }
$$

مبرسشنامه GDS را اولين بار يساواج طراحى كرد و شامل

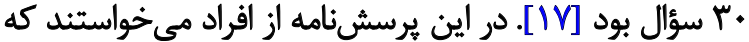

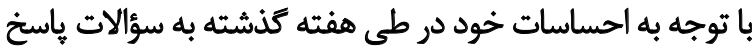

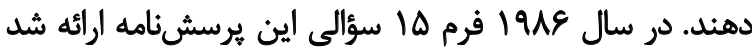

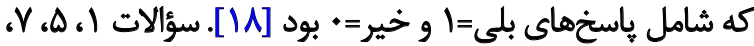

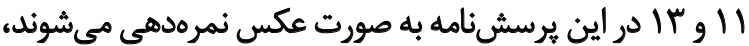

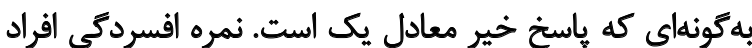

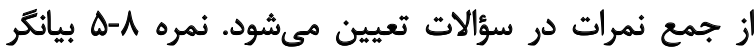

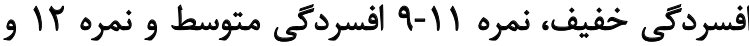

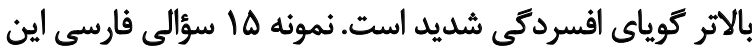

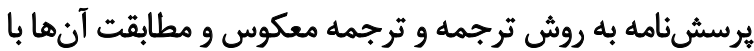

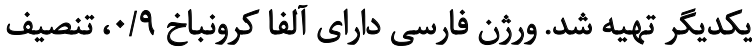

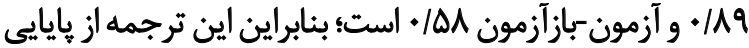

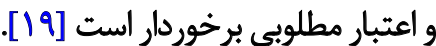

يافتهها

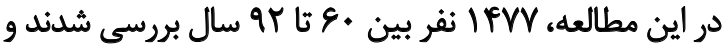

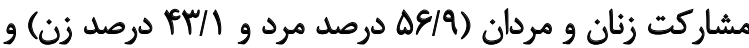

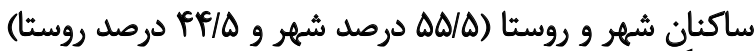

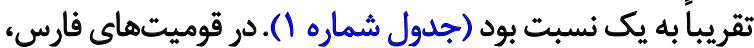

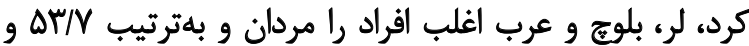
ه ه درصد سالمندان ترك و تركمن مورد مطالعه را زنان تشكيل

1. The Geriatric Depression Scale (GDS-15)
[9]. عوامل مختلفى بر افسردگى سالمندان تأثير كذارند؛ داغداري،

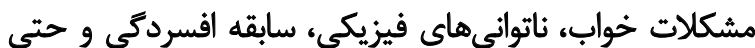

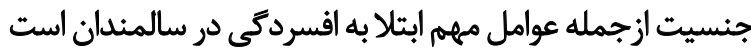

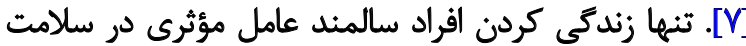

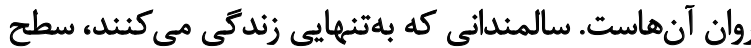

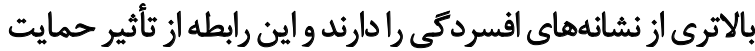

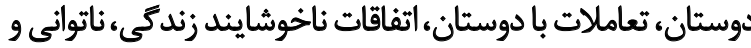

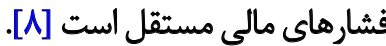
طبق مطالعات انجامشده داخلى و خارجى شيوع افسردگى

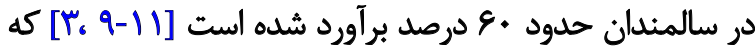

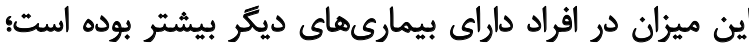

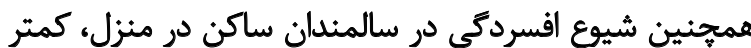

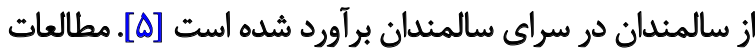

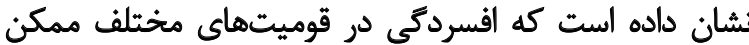

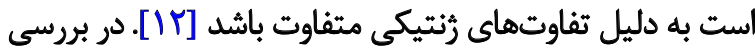

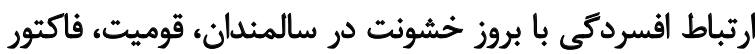

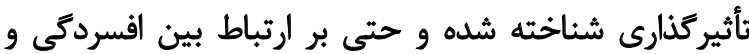

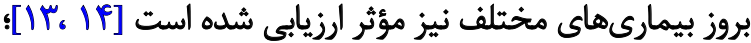

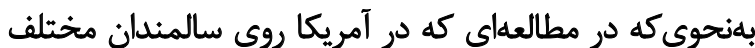

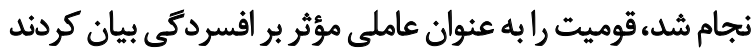

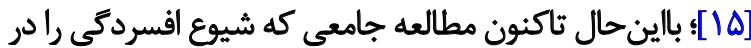

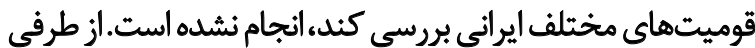

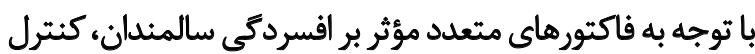

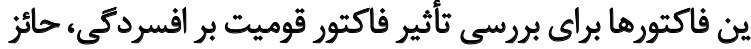

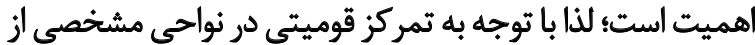

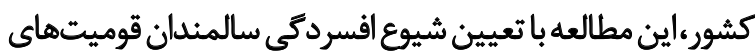

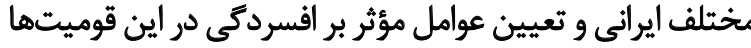

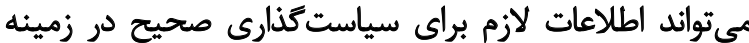
افسردگى سالمندان در مناطق مختلف كشور را فراهم آورد.

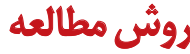

با توجه به اطلاعات هشتمين كنكره بينالمللى رئويوليتيك

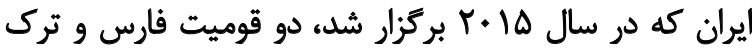

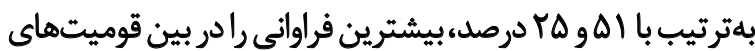

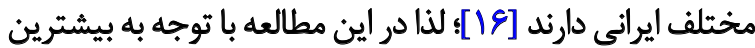

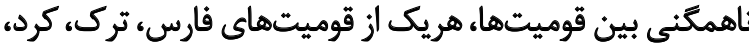

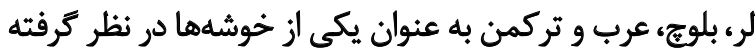

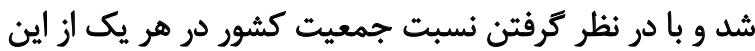

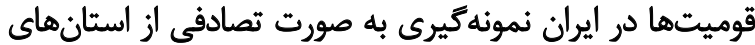

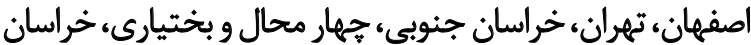

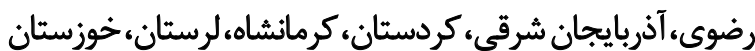

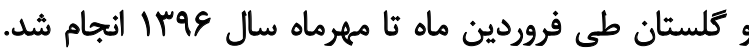

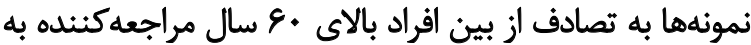

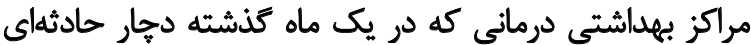


جدول ا. وضعيت متغيرها در سالمندان قوميتهاي مختلف مورد مطالعه

\begin{tabular}{|c|c|c|c|c|c|c|c|c|c|}
\hline \multicolumn{8}{|c|}{ تعداد (درصد) / ميانكين_|نحراف معيار } & \multirow{2}{*}{\multicolumn{2}{|c|}{ قوميت }} \\
\hline جمع & تركمن & 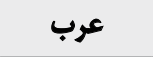 & بلوع & J & كرد & ترك & فارس & & \\
\hline $\operatorname{mr}(1 E / 4)$ & $\Delta(\Delta)$ & $\pi(\pi / \pi)$ & $r q(r q / 4)$ & $r \Delta(r E)$ & $\mathbb{I P}(1 P)$ & $p \cdot(I r / p)$ & $q(1 F)$ & 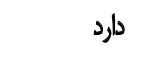 & وضعيت خانه \\
\hline $\mathbb{I T H}(A T / 9)$ & $9 \Delta(9 \Delta)$ & $V \in(V \in / A)$ & $8 \cdot(8 \cdot 19)$ & $n(m)$ & $N(N)$ & $\operatorname{ran}(N \in \mid C)$ & $\Delta \wedge q(N)$ & 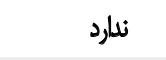 & نتشينى \\
\hline $\operatorname{rar}(\operatorname{mr} / A)$ & Ir(IT) & $\operatorname{Pf}(T+/ F)$ & $F \Delta(F \Delta / \Delta)$ & $\operatorname{Ir}(\mathbb{H} / \Delta)$ & $M(M)$ & $\operatorname{Rq}(\mathrm{rg} / \mathrm{A})$ & $\operatorname{IRT}(r+M)$ & مجرد & \\
\hline IITA (NE/T) & $M(M)$ & $\Delta \Delta(\Delta \Delta / g)$ & Qf $(\Delta f / \Delta)$ & $A f(A V / \Delta)$ & $\operatorname{Ar}(A T)$ & $M 19(n / \Delta)$ & $\Delta R T(V R T)$ & متأهل & \\
\hline $\operatorname{reg}(M)$ & $r(V)$ & $r(r)$ & $n \in(M \in M)$ & $\mathbb{I r}(M / \Delta)$ & $r(V)$ & $\Delta E(N A)$ & $1 \cdot V(1 \otimes / 9)$ & $r+.<$ & \\
\hline rAq (IVq) & $10(10)$ & $\operatorname{PA}(F N \Delta)$ & $1+(1+/ 1)$ & $19(191)$ & $\operatorname{Ir}(I V)$ & $\operatorname{Ar}(\mathrm{rVT})$ & $9 T(1 H / 9)$ & $r \cdot .+F_{* *}$ & سطح درآمد \\
\hline$r \cdot q(r \cdot / q)$ & $M(r)$ & $W(M V / T)$ & $\Lambda(N)$ & 19 (IVA) & $m(r)$ & $R(T V / \Delta)$ & $119(1 \mathrm{~V} / \mathrm{t})$ & $f \cdot \cdot-A_{0}$. & (تومانو) \\
\hline $\operatorname{eir}(f) /(\Delta)$ & PV(PV) & $\|(11 / 1)$ & $V(M / 1)$ & $P f(F V / q)$ & 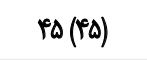 & $91(T \cdot \Delta)$ & $\operatorname{rgs}(\Delta t / p)$ & $\wedge \cdot \cdot>$ & \\
\hline $\operatorname{rqv}(T \cdot / 1)$ & $M(M)$ & $q(8 / 1)$ & $\mathbb{I r}(\mid r /)$ & $\Pi(\Pi \pi / M)$ & $m e(m)$ & A) $(\pi / T)$ & $\| f(18 / 9)$ & شاغل & وضويت \\
\hline $1114 \cdot($ VVq) & $\operatorname{Ar}(A T)$ & $q \pi(q \% / q)$ & $\operatorname{Ar}(A V / Q)$ & $\mathscr{R}(F / M)$ & $\Leftrightarrow(99)$ & $\operatorname{rir}(N / A)$ & $\Delta V(A r / f)$ & بلإئشسته & الشتثال \\
\hline $\operatorname{gre}(9+/ 1)$ & $\Delta \Delta(\Delta \Delta)$ & $\left.p+(p+/)^{+}\right)$ & $P T(P T / N)$ & $\pi(\mu \varphi / \%)$ & $r \cdot(r+)$ & $18 \cdot(\Delta T / V)$ & $\operatorname{rra}(\varphi+1)$ & ij & \\
\hline$A F)(\Delta S / q)$ & $P \otimes(f \otimes)$ & $\Delta q(\Delta V E)$ & $\Delta(\omega / C)$ & $g(F \Delta / E)$ & $V \cdot(V \cdot)$ & IrA(FE/M) & $p) \cdot(\Delta Q q)$ & مرد & جنسي \\
\hline $\operatorname{s\Delta \Lambda }(\mu \oplus / \Delta)$ & $P(P V)$ & $A Y(A Y / q)$ & 19 (198) & $E \Delta(F V / M)$ & $1 .(1)$. & $1 \pi m(P \varphi / 9)$ & $m r(r+1)$ & روستا & \\
\hline $119(\Delta \Delta / \Delta)$ & $\Delta H^{\prime}\left(\Delta Y^{\prime}\right)$ & $\mathbb{I r}(I r /)$ & $1 \cdot(1 . / 1)$ & $M(M T / \Psi)$ & $q \cdot(q \cdot)$ & $1 \notin \Delta(\Delta \mathrm{D} / \mathrm{H})$ & $\operatorname{PAn}(E \subset / q)$ & شير & \\
\hline SQREIVIFE & \&V/Ar $\pm \varnothing 1 . \varnothing$ & VV/ANEV/ar & $n^{m / 1} \Psi \pm N \cdot \Gamma$ & sele. \pm elet & $q x / q 4 \pm \Delta / A Y$ & $E N Y+ \pm V / M$ & $V \cdot 1 \cdot r \pm V / T \pi$ & & \\
\hline$r / \& \cdot \pm V / \Delta T^{r}$ & $r / q . \pm 1 / / T$ & $P /|F \pm Y / A|$ & $T / T E \pm V / P T$ & $r / N q \pm r / / r$ & T/DFE $\pm / A Y$ & Y/TEEV/.1 & $r / T I \pm \cdot / q T$ & كخانواده & تعلداد| \\
\hline$V / T A \pm V / T I$ & VTa $\pm V \cdot a$ & $r / M \pm V / \cdot r$ & I/TQ $\pm / / m$ & $V / \gamma \pm V / 10$ & Vort $/ / Y$ & V/RTEV/I9 & $V / r q \pm 1 / r$ & كاي مزمن & تعلاد بيي \\
\hline
\end{tabular}

جنسيت، محل زندكى، تعداد بيمارىهاى مزمن و قوميت افراد

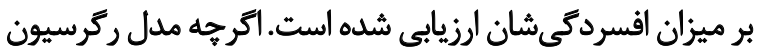

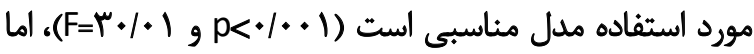

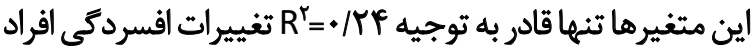

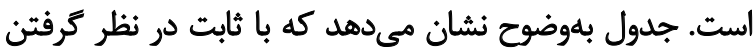

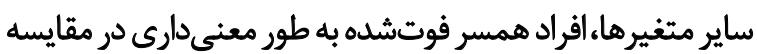

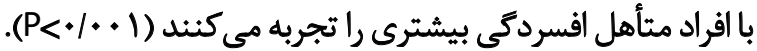

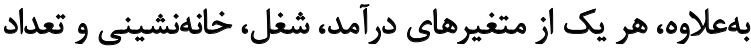

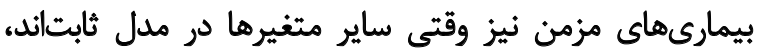

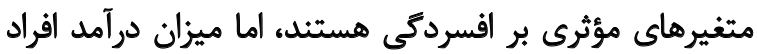

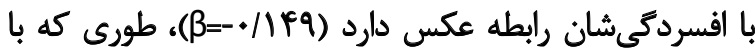

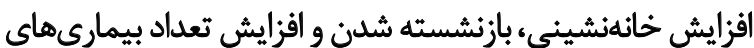

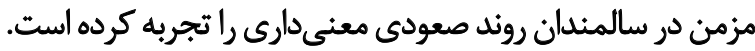

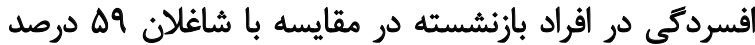

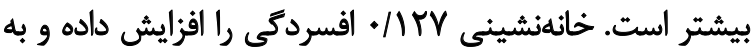

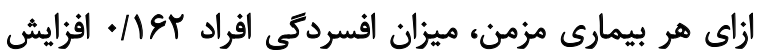

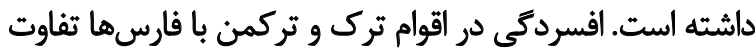
معنى الرى ندارد (

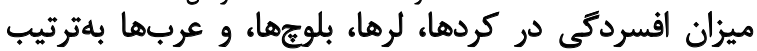

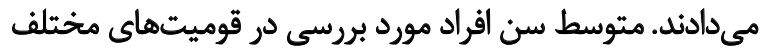

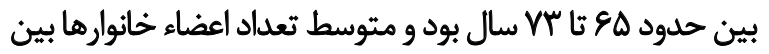

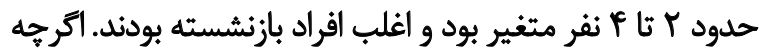

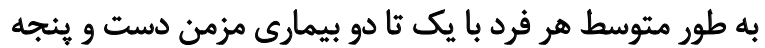

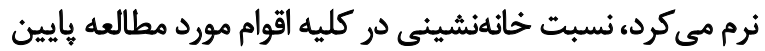

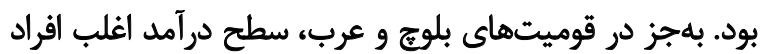

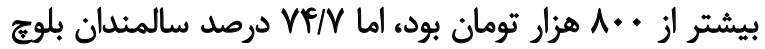

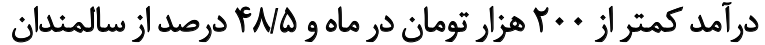

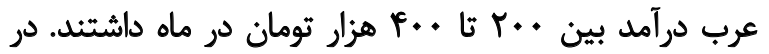

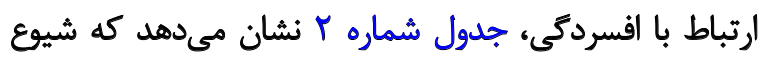

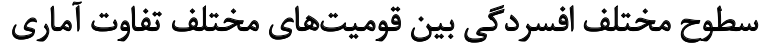

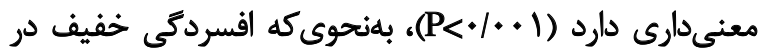

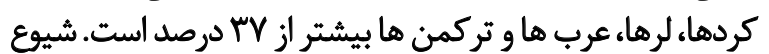

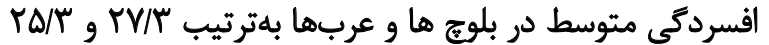

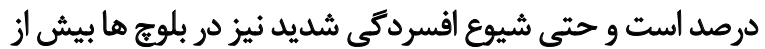

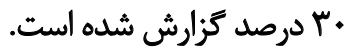

در جدول شماره ب تأثير متغيرهاى سن، وضعيت تأهل، تعداد

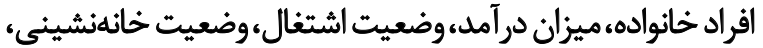


جدول r. مقايسه شيوع شدتهاي مختلف افسردكى در بين اقوام مختلف ايرانى

\begin{tabular}{|c|c|c|c|c|c|}
\hline \multirow{2}{*}{ آماره آزمون كاي اسكوئر } & \multicolumn{4}{|c|}{ تعداد (درصد) } & \multirow{2}{*}{ قُت أفسردكئى } \\
\hline & شيديد & متوسط & خفيف & ندارد & \\
\hline \multirow{7}{*}{$\left.19 V / 1 f(<+1+*)^{*}\right)$} & $\mu(\Delta / \Delta)$ & $V e(1 / M)$ & $\operatorname{lqV}($ TNA) & $T^{f}(\Delta F / \varepsilon)$ & فارس \\
\hline & $M F(N)$ & $8)(r \cdot 10)$ & $8 \Delta(r / / \Lambda)$ & $1 F \lambda(F q / V)$ & ترى ت ت \\
\hline & if (If) & $9(9)$ & $r q(p q)$ & $\mu \wedge(\mu \Lambda)$ & كرد \\
\hline & $f(f / T)$ & IV $(\mathrm{IV} / \mathrm{V})$ & $r e(r v / \Delta)$ & $p q(f+19)$ & لر ل ال \\
\hline & $w+\left(r+/ \mu^{w}\right)$ & $r Y(T Y / T)$ & $M \in(M \in / T)$ & $M(W Y)$ & بلوج \\
\hline & $f(F)$ & $r \Delta(T \Delta / T)$ & $F \wedge(F N \Delta)$ & $M(T Y / T)$ & عرب \\
\hline & $\cdot(\cdot)$ & $V(V)$ & er (pr) & if (ie) & تركمن \\
\hline
\end{tabular}

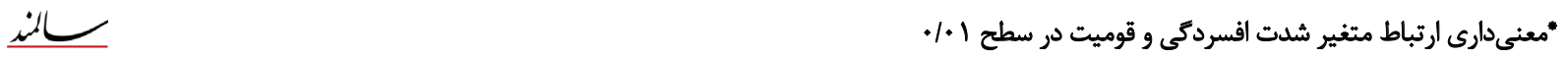

جدول ץ. نتايج آناليز ركرسيون براى متغيرها

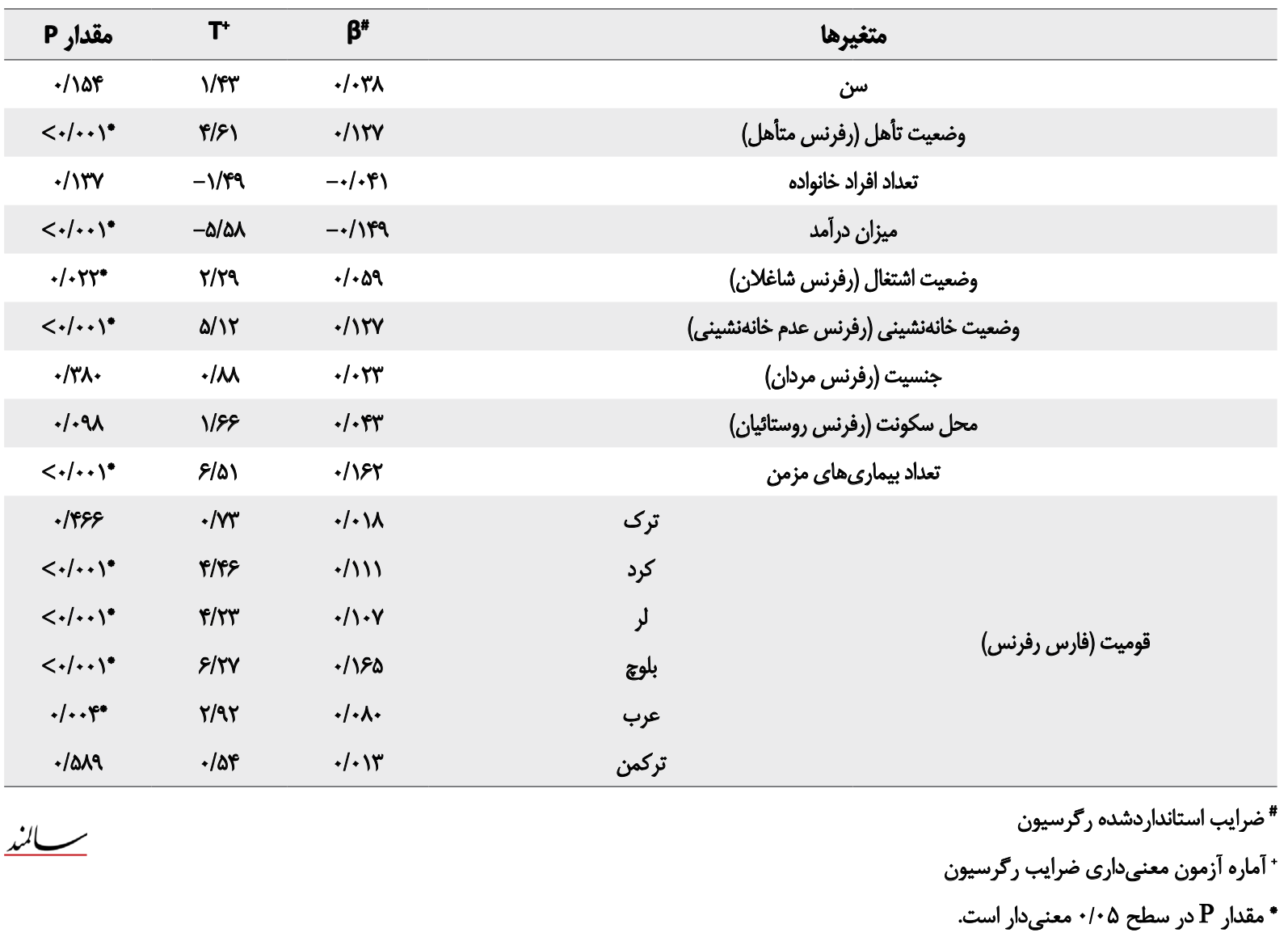

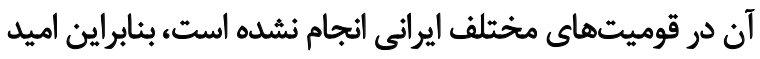

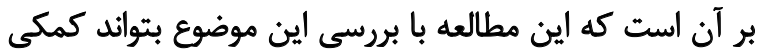

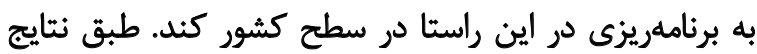

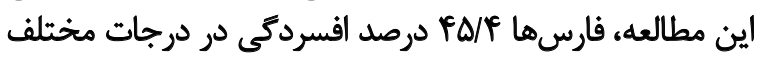

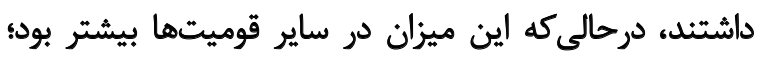

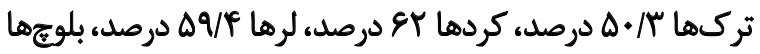
N د درصد، عربها SVI/A

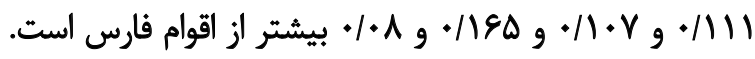
جنسيت، محل زندكَى، سن و حضور افراد ديكر در خانواده فرد

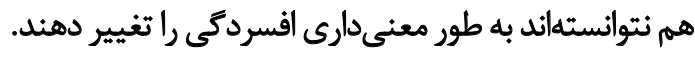
ç? تاكنون مطالعهاي به منظور تعيين شيوع افسردگى و مقايسه 


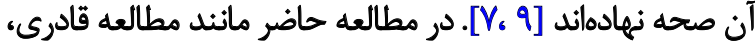

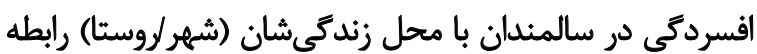

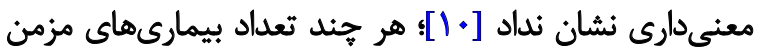

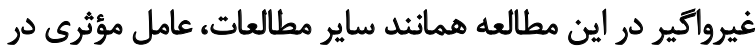

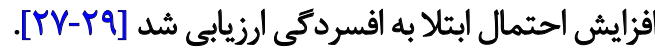
در مطالعهاى مرورى بيان شده است كه تنهايى، عامل خطر ديكر

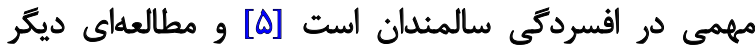

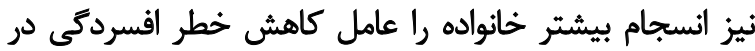

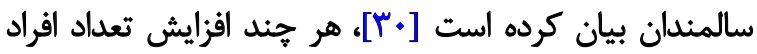

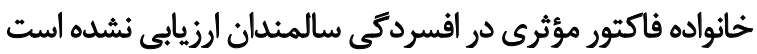
[ [ []. يافتههاى مطالعه ما نيز مؤيد اين مطلب است.

اكر جه حجم نمونه بالا و نمونه ميرى از اغلب نقاط كشور و

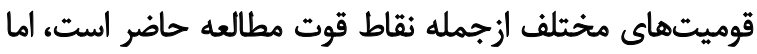

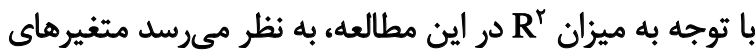

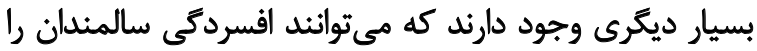

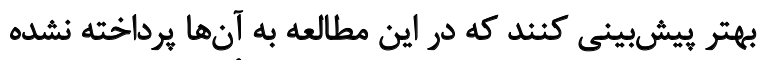

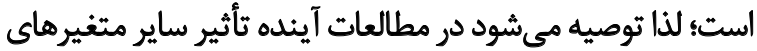

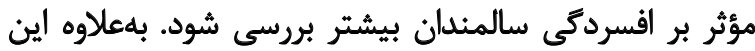

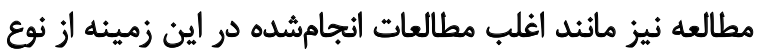

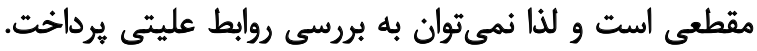

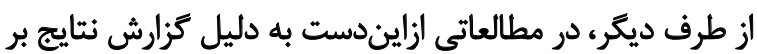

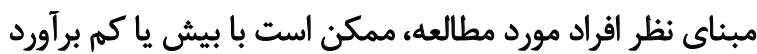

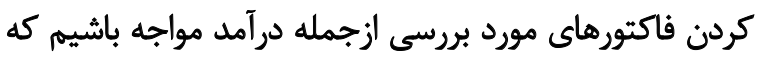

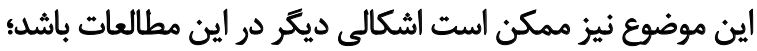

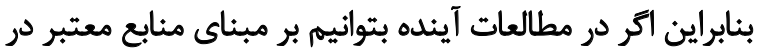

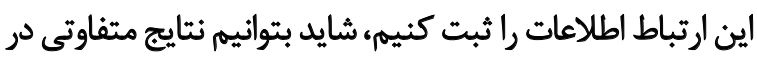

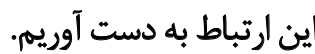

\section{نتيجه ئيرى نهايى}

بر اساس نتايج بهدستآمده از اين تحقيق، تمركز روى موضوع

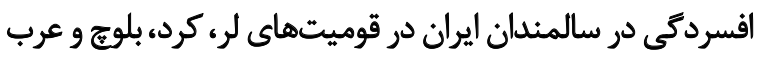

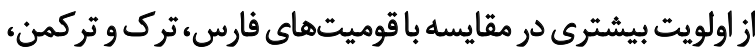

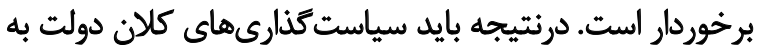

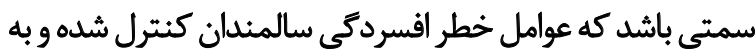

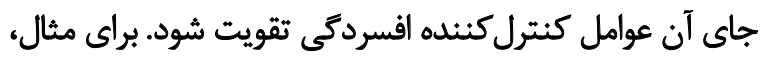

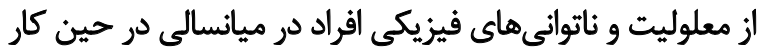

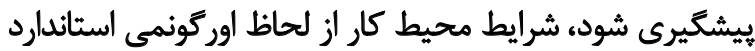

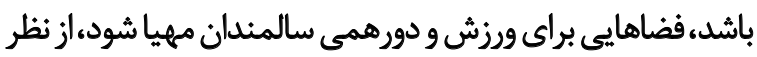

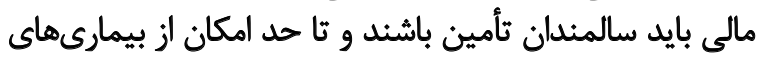

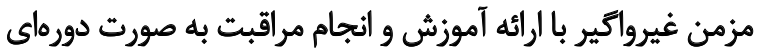

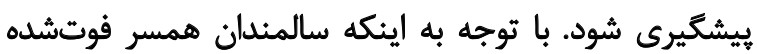

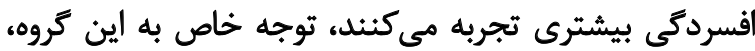

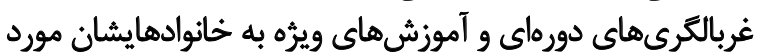

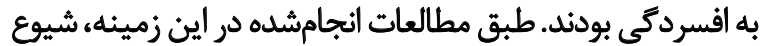

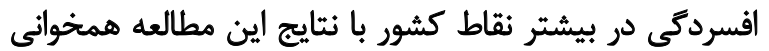

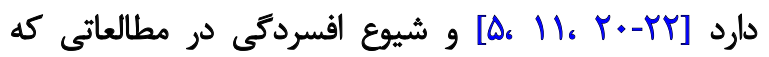

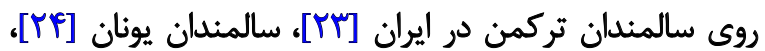

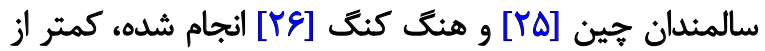

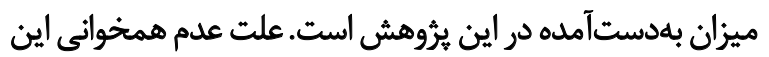

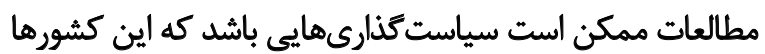

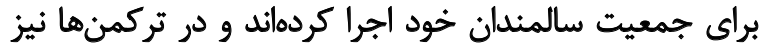

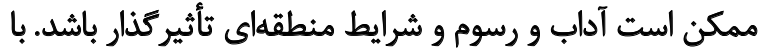

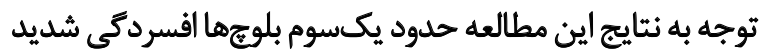

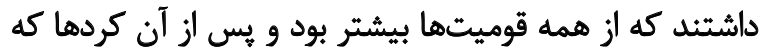

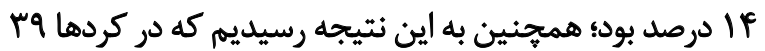

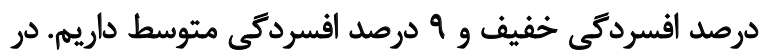

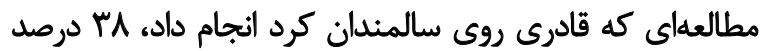

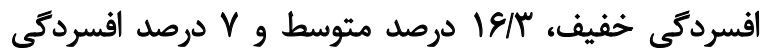

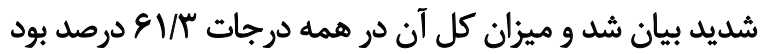

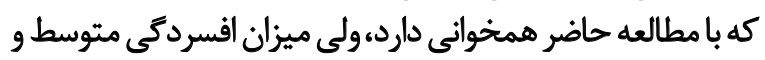

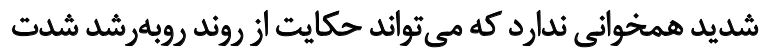

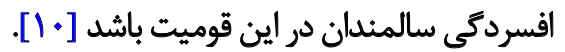

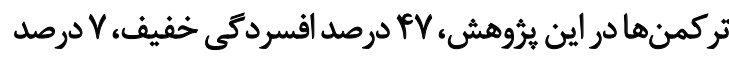

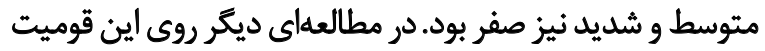

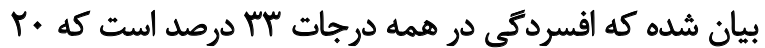

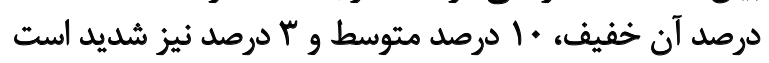

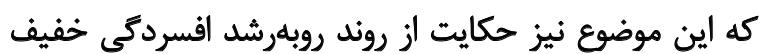

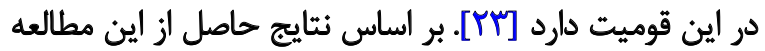

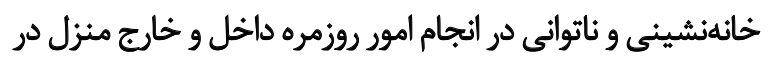

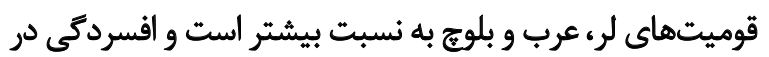

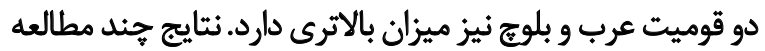

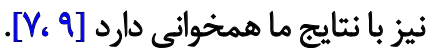

در بين فاكتورهاى مؤثر بر افسردگى سالمندان، وضعيت تأهل،

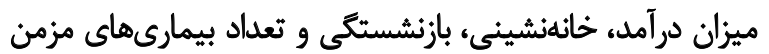

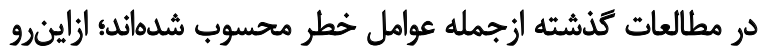

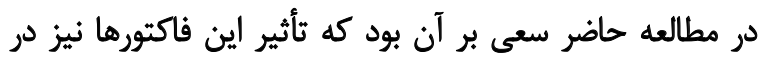

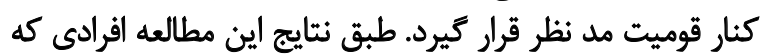

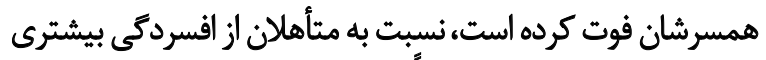

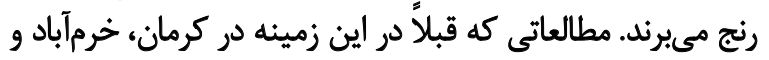

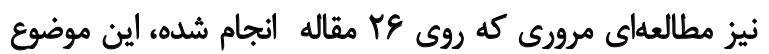

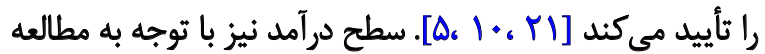

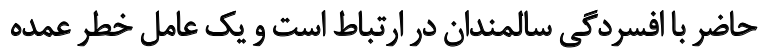

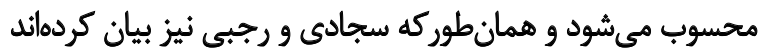

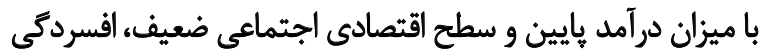

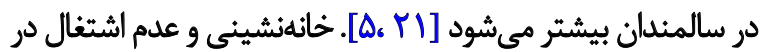

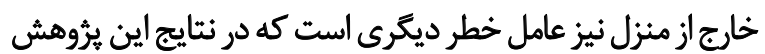

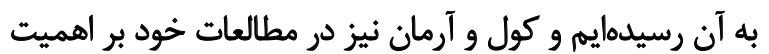


نياز است. متغير ديكرى كه نياز به توجه خاص در برنامهريزي هاي

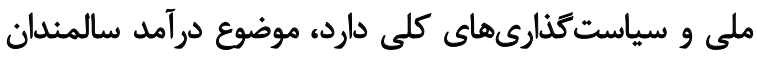

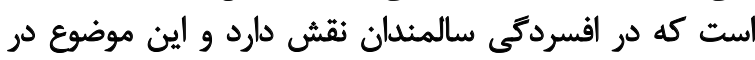

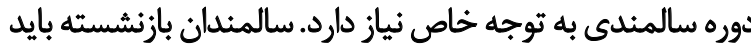

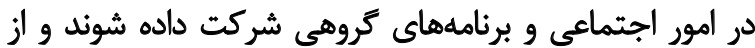

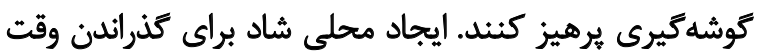
سالمندان نيز مىتواند در اين راستا مؤثر باشد.

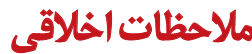

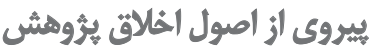

در ايـن مقاله رعايـت شـــه اسـت. شـركت كنيندكان اجـازه

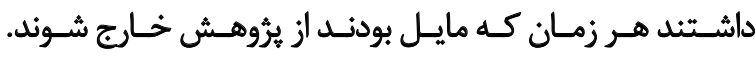

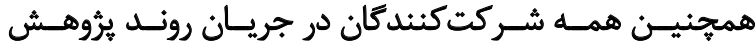

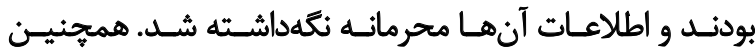

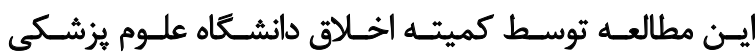

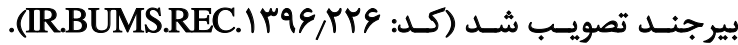

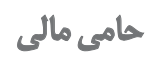

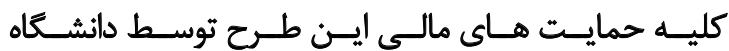

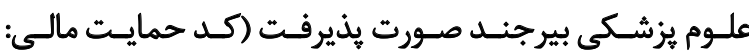
(fF)f

$$
\text { مشار كت نويسند مَّان }
$$

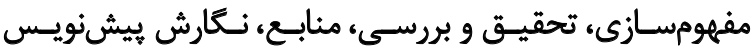

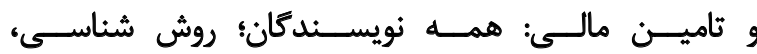

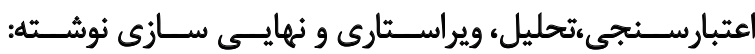

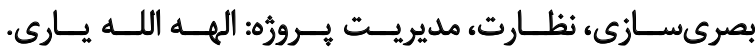

$$
\text { تعارض مثافع }
$$

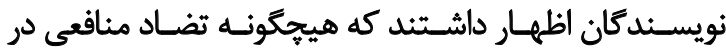
رابطـهـ بـا انتشــار ايسن مقالـه ندارند.

$$
\text { تشيكر و قدرواني }
$$

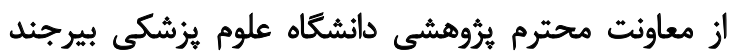

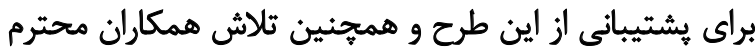

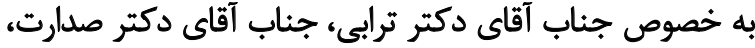

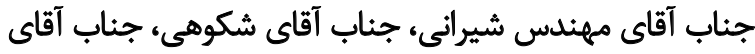

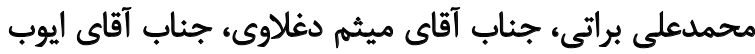

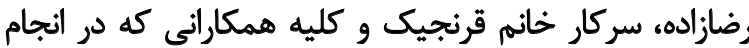

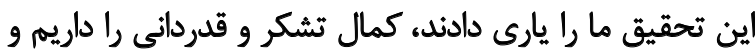

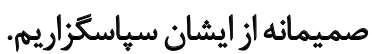




\section{References}

[1] Group W. Study protocol for the World Health Organization project to develop a Quality of Life assessment instrument (WHOQOL) Quality of life Research. 1993; 2(2):153-9. [DOI:10.1007/BF00435734]

[2] Rashidi-Maybodi F, Haerian-Ardakani A, Pour MZ, HeydariPostakan R, Pourbaferani H. Evaluation of Oral Health of Elderly Patients Referring to Khatam ol Anbia Clinic in Yazd in 2014. Arumshealth. 2016; 7(2):227-35.

[3] Lili K. Investigating Hearing Impairment in the Elderly Population of Rural Areas of Rasht City, North of Iran. Journal of Guilan University of Medical Sciences. 2015; 24(94):24-8.

[4] Lashkarboloki F, Aryaei M, Djazayery A, Eftekhar-Ardebily H, Minaei M. Association of demographic, socio-economic features and some health problems with nutritional status in elderly. Iranian Journal of Nutrition Sciences \& Food Technology. 2015; 9(4):27-34

[5] Sajadi H, Mohaqeqi Kamal H, Vameghi M, Forozan AS, Rafei H, Nosratabadi M. Systematic review of prevalence and risk factors associated with depression and its treatment in Iranian elderly. Iranian Journal of Ageing. 2013; 7(4):7-15.

[6] Ahmadi R, Asgary V. Evaluation of individual characteristics, common clinical signs and diet history in patients with hypo- or hyperthyroidism in Hamedan Daneshvar Medicine. 2012; 19(100):37-48.

[7] Cole MG, Dendukuri N. Risk factors for depression among elderly community subjects: a systematic review and meta-analysis. American Journal of Psychiatry. 2003; 160(6):1147-56. [DOI:10.1176/appi. ajp.160.6.1147] [PMID]

[8] Dean A, Kolody B, Wood P, Matt GE. The influence of living alone on depression in elderly persons. Journal of Aging and Health. 1992; 4(1):3-18. [DOI:10.1177/089826439200400101]

[9] Arman M. The Comparison of Depression, Anxiety and Stress between Active and Inactive Old Women in Isfahan. Rehabilitation Medicine. 2014; 3(3):83-8.

[10] Ghaderi S, Sahaf R, Mohammadi Shahbalaghi F, Ansari G, Gharanjic A, Ashrafi K, et al. Prevalence of depression in elderly Kurdish community residing in Boukan, Iran. Iranian Journal of Ageing. 2012; 7(1):57-66.

[11] Mirzaei M, Sahaf R, Mirzaei S, Sepahvand E, Pakdel A, Shemshadi H. Depression and its associated factors in elderly nursing home residents: A screening study in Khorramabad. Iranian Journal of Ageing. 2015; 10(1):54-61.

[12] Minallah A, Azam N, Merani I. Frequency of depression with associated risk factors among elderly in two tertiary care hospitals in rawalpindi. Pakistan Armed Forces Medical Journal. 2019; 69(2):317-21.

[13] Narendran M, Kumar DS, Kulkarni P, Renuka M, Murthy N. Treat the troika: does depression and malnutrition affect activities of daily living? A study among elderly soliga tribes, br hills, karnataka. Indian Journal of Public Health Research \& Development. 2019; 10(5):53-8. [DOI:10.5958/0976-5506.2019.00968.9]

[14] Pereira JA, Mendes F, Mestre T, Zangão MO, Pereira C. The Relationship Between Depression and Violence Risk Predictors on Elderly. International Journal of Studies in Nursing. 2018; 4(1):9-19. [DOI:10.20849/ijsn.v4i1.536]

[15] Leet JE. Benefits of Prayer on Depression in Elderly Adults. Minnesota: Walden University; 2018.
[16] Salehabadi R, Janparvar M, editors. Opportunities for Ethnic Presence in the Islamic Republic of Iran. Eighth Congress of the Iranian Geopolitical Society entitled: Empathy for Iranian Ethnic National Cohesion and Authority; 2015.

[17] Yesavage JA, Sheikh JI. 9/Geriatric depression scale (GDS) recent evidence and development of a shorter version. Clinical gerontologist. 1986; 5(1-2):165-73. [DOI:10.1300/J018v05n01_09]

[18] Lyness JM, Noel TK, Cox C, King DA, Conwell Y, Caine ED. Screening for depression in elderly primary care patients: A comparison of the Center for Epidemiologic Studies-Depression Scale and the Geriatric Depression Scale. Archives of Internal Medicine. 1997; 157(4):449-54. [DOI:10.1001/archinte.1997.00440250107012] [PMID]

[19] Malakouti K, Fathollahi P, Mirabzadeh A, Salavati M, Kahani S. Validation of Geriatric Depression Scale (GDS-15) in Iran. Research in Medicine. 2006; 30(4):361-9.

[20] Manzouri L, Babak A, Merasi M. The depression status of the elderly and it's related factors in Isfahan in 2007. Iranian Journal of Ageing. 2010; 4(4):0-.

[21] Rajabizadeh GH, Ramezani A. Prevalence of depression in kermanian geriatrics - 2002 (1381). Journal of Rafsanjan University of Medical Sciences and Health Services. 2004; 3(1):58-66.

[22] Alipour F, Sajadi H, Forouzan A, Nabavi H, Khedmati E. The role of social support in the anxiety and depression of elderly. Iranian Journal of Ageing. 2009; 4(1):0-

[23] Gharanjik A, Mohammadi Shahbolaghi F, Ansari G, Najafi F, Ghaderi S, Ashrafi K, et al. The prevalence of depression in older Turkmen adults in 1389. Iranian Journal of Ageing. 2011; 6(3):34-9.

[24] Papadopoulos F, Petridou E, Argyropoulou S, Kontaxakis V, Dessypris N, Anastasiou A, et al. Prevalence and correlates of depression in late life: A population based study from a rural Greek town. International Journal of Geriatric Psychiatry. 2005; 20(4):350-7. [DOI:10.1002/gps.1288] [PMID]

[25] Gao S, Jin Y, Unverzagt FW, Liang C, Hall KS, Ma F, et al. Correlates of depressive symptoms in rural elderly Chinese. International Journal of Geriatric Psychiatry. 2009; 24(12):1358-66. [DOI:10.1002/ gps.2271] [PMID] [PMCID]

[26] Chi I, Yip PS, Chiu HF, Chou KL, Chan KS, Kwan CW, et al. Prevalence of depression and its correlates in Hong Kong's Chinese older adults. The American journal of geriatric psychiatry. 2005; 13(5):409-16. [DOI:10.1097/00019442-200505000-00010] [PMID]

[27] Alexopoulos GS. Depression in the elderly. The Lancet. 2005; 365(9475):1961-70. [DOI:10.1016/S0140-6736(05)66665-2]

[28] Djernes JK. Prevalence and predictors of depression in populations of elderly: A review. Acta Psychiatrica Scandinavica. 2006; 113(5):372-87. [DOI:10.1111/j.1600-0447.2006.00770.x] [PMID]

[29] Saeidimehr S, Geravandi S, Izadmehr A, Mohammadi MJ. Relationship between the "Quality of Life" and symptoms of depression among older adults. Iranian Journal of Ageing. 2016; 11(1):90-9. [DOI:10.21859/sija-110190]

[30] Park M, Unützer J, Grembowski D. Ethnic and gender variations in the associations between family cohesion, family conflict, and depression in older Asian and Latino adults. Journal of immigrant and minority health. 2014; 16(6):1103-10. [DOI:10.1007/s10903-013-99261] [PMID] [PMCID] 